Subscriber access provided by Caltech Library

Communication

\title{
Defect-Seeded Atomic Layer Deposition of Metal Oxides on the Basal Plane of 2D Layered Materials
}

Michael F. Mazza, Miguel Cabán-Acevedo, Joshua D Wiensch, Annelise C. Thompson, and Nathan S. Lewis Nano Lett., Just Accepted Manuscript • DOI: 10.1021/acs.nanolett.0c00179 • Publication Date (Web): 25 Mar 2020

Downloaded from pubs.acs.org on March 25, 2020

\section{Just Accepted}

"Just Accepted" manuscripts have been peer-reviewed and accepted for publication. They are posted online prior to technical editing, formatting for publication and author proofing. The American Chemical Society provides "Just Accepted" as a service to the research community to expedite the dissemination of scientific material as soon as possible after acceptance. "Just Accepted" manuscripts appear in full in PDF format accompanied by an HTML abstract. "Just Accepted" manuscripts have been fully peer reviewed, but should not be considered the official version of record. They are citable by the Digital Object Identifier (DOI®). "Just Accepted" is an optional service offered to authors. Therefore, the "Just Accepted" Web site may not include all articles that will be published in the journal. After a manuscript is technically edited and formatted, it will be removed from the "Just Accepted" Web site and published as an ASAP article. Note that technical editing may introduce minor changes to the manuscript text and/or graphics which could affect content, and all legal disclaimers and ethical guidelines that apply to the journal pertain. ACS cannot be held responsible for errors or consequences arising from the use of information contained in these "Just Accepted" manuscripts. 


\title{
Defect-Seeded Atomic Layer Deposition of Metal Oxides on the Basal Plane of 2D Layered Materials
}

Michael F. Mazza, †¥ Miguel Cabán-Acevedo, $¥$ Joshua D. Wiensch, $†$ Annelise C.

Thompson, t and Nathan S. Lewist

†127-72, 210 Noyes Laboratory, Division of Chemistry and Chemical Engineering,

California Institute of Technology, Pasadena, California 91125, United States

$\ddagger$ These authors contributed equally.

KEYWORDS: Defect driven growth, atomic layer deposition, dislocation networks, 2D layered materials.

\begin{abstract}
Atomic layer deposition (ALD) on mechanically exfoliated 2D layered materials spontaneously produces network patterns of metal oxide nanoparticles in triangular and linear deposits on the basal surface. The network patterns formed under a range of ALD conditions, and were independent of the orientation of the substrate in the
\end{abstract}




\begin{abstract}
ALD reactor. The patterns were produced on $\mathrm{MoS}_{2}$ or HOPG when either tetrakis(dimethylamido)titanium or bis(ethylcyclopentadienyl)manganese were used as precursors, suggesting that the phenomenon is general for $2 \mathrm{D}$ materials. Transmission electron microscopy revealed the presence, prior to deposition, of dislocation networks along the basal plane of mechanically exfoliated 2D flakes, indicating that periodical basal plane defects related to disruptions in the van der Waals stacking of layers, such as perfect line dislocations and triangular extended stacking faults networks, introduce a surface reactivity landscape that leads to the emergence of patterned deposition.
\end{abstract}

\title{
INTRODUCTION
}

2D layered materials, such as graphite and transition metal dichalcogenides $\left(M X_{2}\right.$, where $M=\mathrm{Mo}$ and $\mathrm{W}$, and $X=\mathrm{S}, \mathrm{Se}$, and Te), have strong, covalent in-plane bonding and weak van der Waals interactions between layers. Within the few-layer to monolayer regime, the electronic, chemical, and optical properties of layered materials are dependent on thickness, stacking order, and relative stacking orientation. ${ }^{[1-3]}$ Given the variety of electronic structures and chemistry of $2 \mathrm{D}$ materials, and their tunability at the 
nanoscale, this class of materials has been actively investigated in applications such as catalysis, ${ }^{[4,5]}$ batteries, ${ }^{[6,7]}$ and photovoltaics. ${ }^{[8,9]}$

Pristine basal surfaces on bulk 2D single crystals and/or pristine few-layer stacks of 2D materials commonly used in application-focused studies are generally prepared by mechanical exfoliation with adhesive tape. Transmission electron microscopy (TEM) studies have shown that mechanical exfoliation can disrupt the relative stacking orientation of 2D layers at or within a few layers of the surface of the single crystal. ${ }^{[10]}$ The disruptions caused by the shear and twist forces involved in the mechanical exfoliation process can induce surface corrugation and can also form dislocation networks near the surface and along the basal plane. Mechanical exfoliation can consequently induce defect patterns and networks that modify the local electronic structure and chemical reactivity of the basal plane surface. ${ }^{[11-13]}$

In principle, the local differences in properties induced by defects can consequently alter the chemical reactivity of the surface. Such heterogeneity can be exploited to produce patterned deposition using defect-selective deposition methods and reaction conditions. Atomic layer deposition (ALD) allows excellent thickness control and film 
conformity, and consequently is used to fabricate gate dielectrics as well as protective coatings for photoelectrochemical devices. ${ }^{[14,15]}$ ALD utilizes sequential, self-limiting chemical reactions for the controlled growth of highly conformal films on the surface of a target substrate. Selective-area ALD has been demonstrated by use of an assisting selfabsorbed monolayer to inhibit facile penetration of precursor molecules through the monolayer to the surface of the substrate. ${ }^{[16]}$ Selective ALD has also been reported at boundaries and step edges on the basal plane surface of $2 \mathrm{D}$ layered materials. ${ }^{[17,18]}$ While such studies exploit the high-reactivity of dangling bond and grain boundary defect sites to achieve preferential deposition, an alternative approach would exploit the surface energy landscape introduced by defect networks originating solely from disruption of van der Waals (non-bonding) interactions to produce patterned deposition.

We report herein the spontaneous, unassisted growth of highly ordered triangular arrays of metal oxide particles on the basal surface of mechanically exfoliated molybdenum disulfide $\left(\mathrm{MoS}_{2}\right)$ as well as on the basal plane of highly oriented pyrolytic graphite (HOPG). The ALD reaction conditions and the metal organic precursor were systematically studied to understand the origin and generality of the patterned deposition. 
Additionally, the presence of dislocation networks prior to deposition was investigated by

TEM.

\section{RESULTS AND DISCUSSION}

Effect of Titanium Precursor Dose Time. Figure 1a-f shows scanning electron microscopy (SEM) images for three mechanically exfoliated $\mathrm{MoS}_{2}$ crystals covered with 1000 ALD cycles of titanium dioxide $\left(\mathrm{TiO}_{2}\right)$. The $\mathrm{TiO}_{2}$ was formed using different tetrakis(dimethylamido)titanium (TDMAT) precursor dose times, t(TDMAT), while using water as the counter reactant. For $t(T D M A T)=0.025 \mathrm{~s}$, in certain regions of the basal surface the $\mathrm{TiO}_{2}$ film formed a highly ordered network consisting of triangularly shaped island deposits that shared corners and overall orientation. Very little nanoparticle deposition was observed in the interior of the opposite-facing triangularly shaped empty spaces generated by the $\mathrm{TiO}_{2}$ film pattern (Figure 1d).

The dose time for the metal organic precursor was then systematically increased to investigate the effect that TDMAT concentration per cycle in the ALD chamber had on the $\mathrm{TiO}_{2}$ film growth and on the morphology of the network pattern. Increases in the dose time produce a concomitant increase in the $\mathrm{TiO}_{2}$ film growth-per-cycle (GPC). 
Ellipsometry data indicated a GPC of $0.47 \AA \mathrm{cycle}^{-1}, 0.55 \AA \mathrm{Acycle}^{-1}$, and $0.73 \AA \mathrm{cycle}^{-1}$ for dose times of $0.025 \mathrm{~s}, 0.1 \mathrm{~s}$, and $0.4 \mathrm{~s}$, respectively. The ellipsometry data of the GPC values were in accord with $\mathrm{TiO}_{2}$ film-thickness data determined using atomic-force microscopy (see Supporting Information for more details). As the dose time was increased to $t($ TDMAT $)=0.1 \mathrm{~s}$, the concentration of nanoparticles within the oppositefacing triangularly shaped empty spaces gradually increased. The smoothness and uniformity of the triangularly shaped $\mathrm{TiO}_{2}$ deposition also improved, indicating an increase in the conformality and compactness of the $\mathrm{TiO}_{2}$ film. At $t($ TDMAT $)=0.4 \mathrm{~s}$, the empty spaces were mostly filled with $\mathrm{TiO}_{2}$ nanoparticles. Moreover, these opposite-facing triangularly shaped regions displayed a brighter contrast under SEM, due to the decrease in the conformality and compactness of the film in these regions. 


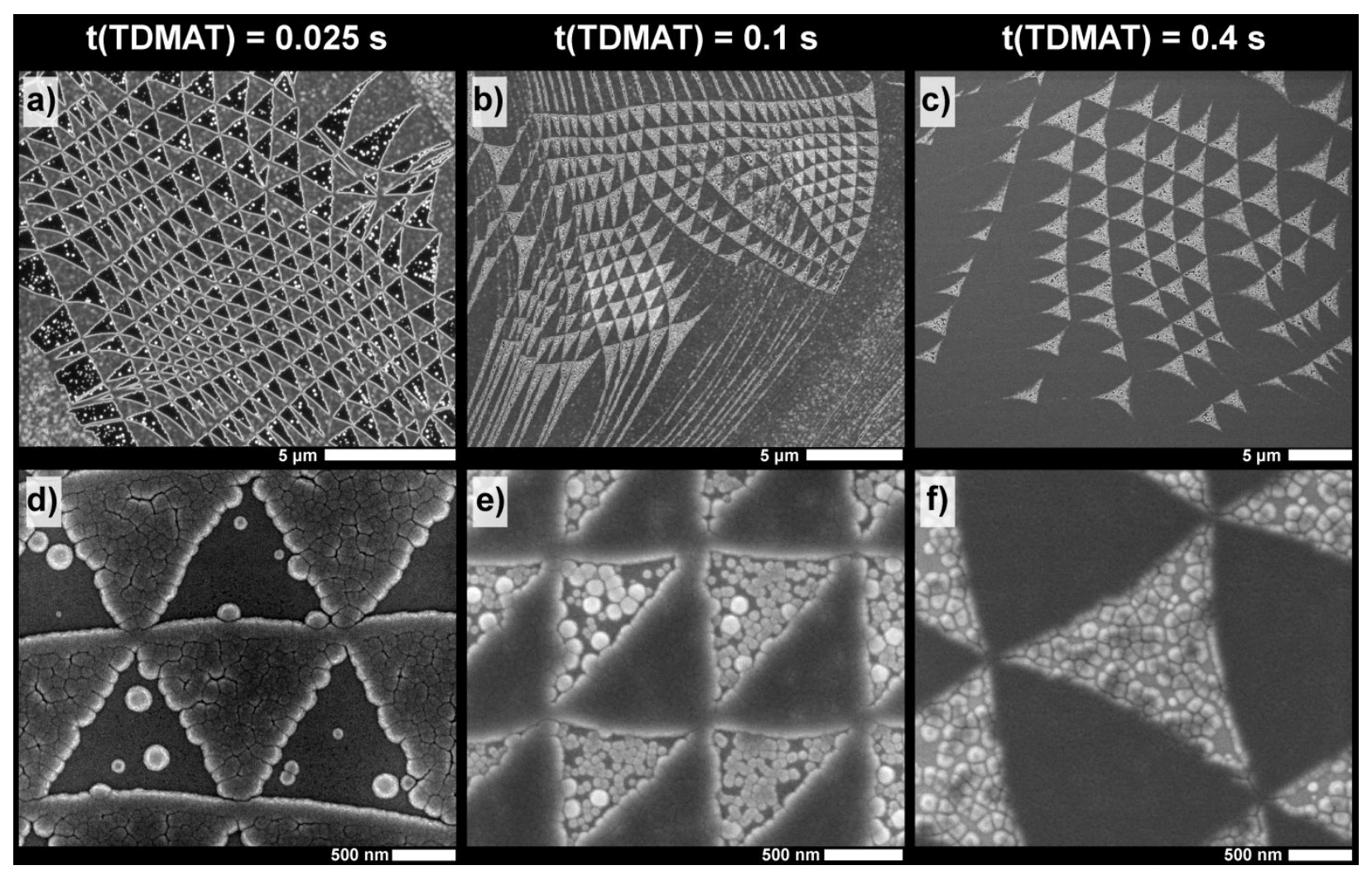

Figure 1. SEM images of patterned regions on $\mathrm{MoS}_{2}$ after 1000 ALD cycles of TDMAT and $\mathrm{H}_{2} \mathrm{O}$ when the pulse time of TDMAT was varied from $(\mathrm{a}, \mathrm{d}) 0.025 \mathrm{~s},(\mathrm{~b}, \mathrm{e}) 0.1 \mathrm{~s}$ and (c,f) $0.4 \mathrm{~s}$ respectively. Scale bars are $5 \mu \mathrm{m}(\mathrm{a}, \mathrm{b}, \mathrm{c})$ and $500 \mathrm{~nm}(\mathrm{~d}, \mathrm{e}, \mathrm{f})$.

The dose time data clearly indicates the emergence of inert, triangular regions on selected areas of the basal plane surface through a range of ALD reaction conditions. Assuming inhomogeneous surface reactivity, at short dose times the low TDMAT concentration shifts the reaction towards a lower supersaturation, more selective condition, and the $\mathrm{TiO}_{2}$ deposition becomes favorable only on the most reactive (high- 
energy) basal surface sites. In contrast, at longer dose times, and correspondingly high growth rates, the increase in the TDMAT concentration shifts the reaction towards a higher supersaturation condition, with deposition of $\mathrm{TiO}_{2}$ resulting in a conformal film outside of these bright triangular regions. The reported linear growth rate for TDMAT and $\mathrm{H}_{2} \mathrm{O}$ at $150{ }^{\circ} \mathrm{C}$ is $\sim 0.52 \AA$ cycle $^{-1} .{ }^{[19]}$ The growth rate at the longest dose time is $50 \%$ higher than expectations, so the random nanoparticle deposition in the empty spaces could indicate fractional CVD-type deposition, which correspondingly increases as the precursor dose time increases. The observed film patterns therefore suggest that the basal surface contains areas where the surface energy is organized in a network pattern comprising a continuous array of two distinct opposite-facing triangularly shaped regions with substantially different reactivities.

Effect of ALD Growth Time. Figure 2a-f shows the evolution of the morphology of the $\mathrm{TiO}_{2}$ film pattern as the total deposition proceeded through 200,400 , and 1000 cycles, respectively, for a fixed exfoliated $\mathrm{MoS}_{2}$ region and a dose time of $t($ TDMAT $)=0.1 \mathrm{~s}$. To probe effects of the direction of the gas flow on the deposition process, after the first 400 cycles the sample was rotated $180^{\circ}$ in the chamber. After 200 cycles $(\sim 10 \mathrm{~nm})$ of growth, 
a $\mathrm{TiO}_{2}$ film pattern was clearly manifested as a bright network of triangularly shaped deposits formed from somewhat discontinuous $\mathrm{TiO}_{2}$ nanoparticles (Figure 2a,b). After 400 total deposition cycles, the packing density of $\mathrm{TiO}_{2}$ particles increased within the triangularly shaped deposits (Figure 2c,d). The directionality of the deposition pattern remained the same despite the rotation of the $\mathrm{MoS}_{2}$ substrate by $180^{\circ}$ between 400 and 1000 ALD cycles, indicating that the orientation of the triangularly shaped deposits was independent of the direction of the gas flow within the ALD chamber. At 1000 total ALD cycles (Figure 2e,f) the smoothness, conformity, and compactness of the previously observed triangularly shaped $\mathrm{TiO}_{2}$ deposits increased substantially. In addition, the empty spaces of opposite-facing triangles were filled with $\mathrm{TiO}_{2}$ particles. Differences in the film density and packing induced a reversal in contrast between the original triangularly shaped deposits and the subsequently filled empty spaces, such that the infilled triangles appeared bright whereas the original triangularly shaped deposits appeared darker in contrast in the SEM images. 


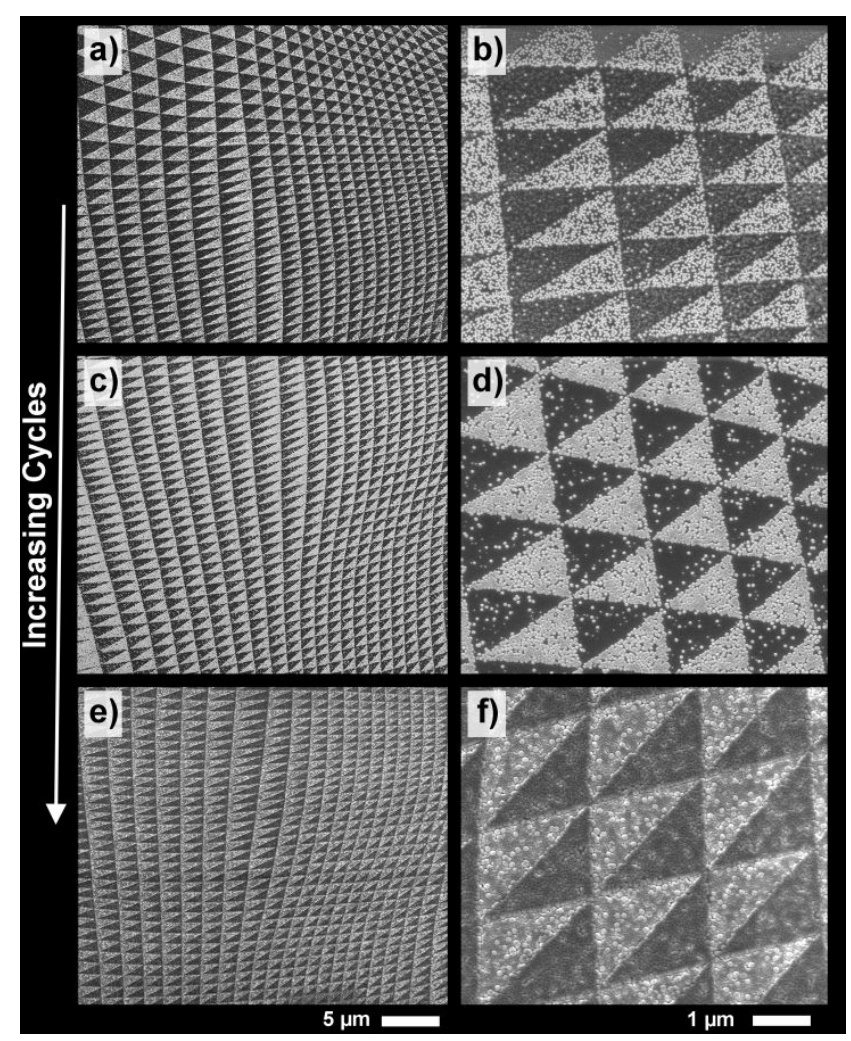

Figure 2. SEM images showing the evolution of a deposition pattern after (a,b) 200, (c,d) 400 , and (e,f) 1000 cycles respectively. After 400 cycles, the sample was rotated $180^{\circ}$ in the ALD chamber to probe for gas directionality effects on the orientation of the deposition. Scale bar is $5 \mu \mathrm{m}$ for the left column and $1 \mu \mathrm{m}$ for the right column.

At a fixed ALD condition, inhomogeneities in the deposition rate are indicative of differences in reactivity among surface sites on the basal plane. The sequential deposition experiment indicates that some areas within the basal surface of mechanically exfoliated 
$\mathrm{MoS}_{2}$ contain a network arrangement of two opposite-facing triangularly shaped regions that have mutually distinct deposition rates. Defects can introduce areas with modified densities of states and reactivities, so the emergence of the observed $\mathrm{TiO}_{2} \mathrm{ALD}$ pattern is consistent with the presence of an underlying defect network.

Effects of ALD Precursor and 2D Substrate on Film Growth. Figure 3 shows the presence of triangularly shaped deposition networks after ALD on mechanically exfoliated $\mathrm{MoS}_{2}$ and HOPG, when either TDMAT or bis(ethylcyclopentadienyl)manganese $\left((\mathrm{EtCp})_{2} \mathrm{Mn}\right)$ were used as metal organic precursors. Each deposition was performed at $150{ }^{\circ} \mathrm{C}$ with water as the counter reactant. The ALD reaction conditions were tuned to access a low growth-rate regime, facilitating selective deposition of the network pattern (methodology section in the Supporting Information). ALD with TDMAT on HOPG (Figure 3c) produced triangularly shaped deposition networks of nominally identical morphology to those produced on $\mathrm{MoS}_{2}$ (Figure 3a). The underlying mechanism enabling the formation of the patterns is thus independent of the specific bonding chemistry of the basal surface, suggesting that formation of the patterns is likely a general characteristic of 2D layered materials. Consistently, triangular networks with morphologies nominally 
identical to those produced on $\mathrm{MoS}_{2}$ and HOPG using TDMAT were observed after ALD using $(E t C p)_{2} \mathrm{Mn}$ on either $\mathrm{MoS}_{2}$ or HOPG (Figure 3b,d). The observation of spontaneous network formation by manganese oxide $\left(\mathrm{MnO}_{\mathrm{x}}\right)$ particles on the surface of mechanically exfoliated $2 \mathrm{D}$ materials strongly suggests that the mechanism of pattern formation is independent of the chemistry and structure of the metal organic precursor.

The inert nature of the pristine basal surface of 2D materials caused by the absence of dangling bonds hinders the deposition of films by ALD by preventing the facile adsorption of precursor molecules. ${ }^{[20,21]}$ Bonding related defects, such as vacancies, act as highenergy sites facilitating precursor surface absorption and enhancing reactivity for deposition. However, bonding related defects within a single basal surface are random in nature and cannot explain the emergence of ordered deposits. In contrast, translational and rotational deformations to the van der Waals stacking of 2D layers induced by mechanical exfoliation can introduce local modifications to the stacking order that manifest as organized dislocation networks. ${ }^{[10]}$ Dislocation networks caused by stacking order disruptions have been shown to modify the density of states, electrical conductivity, photoluminescence, and electronic structure of the layered material. ${ }^{[11,22-24]}$ Thus, the 
presence of dislocation networks could introduce periodic regions with modified surface energy generating a reactivity landscape on the basal surface. The presence and morphology of dislocation networks on $\mathrm{MoS}_{2}$ was therefore investigated to test the hypothesis that the triangularly shaped deposition network pattern on $2 \mathrm{D}$ layered materials resulted from the presence of defect networks in the exfoliated substrates.

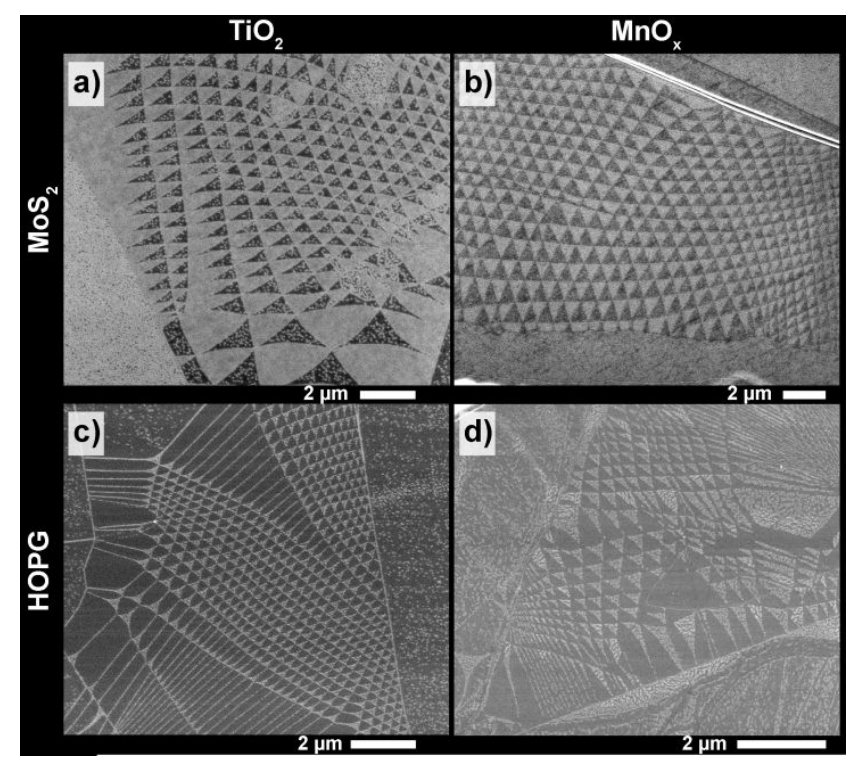

Figure 3. SEM images showing pattern development with different layered substrates and metal oxide precursors. A) The deposition of $\mathrm{TiO}_{2}$ from TDMAT on $\mathrm{MoS}_{2}$. B) $\mathrm{MnO}_{\mathrm{x}}$ on $\mathrm{MoS}_{2}$ from (EtCp) $\left.)_{2} \mathrm{Mn} . \mathrm{C}\right) \mathrm{TiO}_{2}$ on HOPG. D) $\mathrm{MnO}_{\mathrm{x}}$ on HOPG. 


\section{TEM Characterization of Dislocation Networks. Figure 4a shows an SEM image of a}

highly ordered line-network pattern of $\mathrm{TiO}_{2}$ deposits formed by ALD on $\mathrm{MoS}_{2}$ via the recipe

used in Figure 3a. The SEM pattern closely resembled the line pattern of dislocation networks observed by TEM on a mechanically exfoliated $\mathrm{MoS}_{2}$ flake (Figure 4b). The resemblance between the $\mathrm{TiO}_{2}$ deposition pattern and the dislocation network pattern suggests that line defects generated by the disruptions in the stacking orientation of $2 \mathrm{D}$ layers during mechanical exfoliation can seed deposition under a range of low supersaturation conditions, and thus act similarly to the seeding effect of bonding-related defects. By analogy to previous studies on bonding-related defects, ${ }^{[25]} 2 \mathrm{D}$ stackingrelated defects can therefore also serve as high-energy sites that lower the activation energy for heteronucleation, enabling preferential deposition under low supersaturation 
conditions.

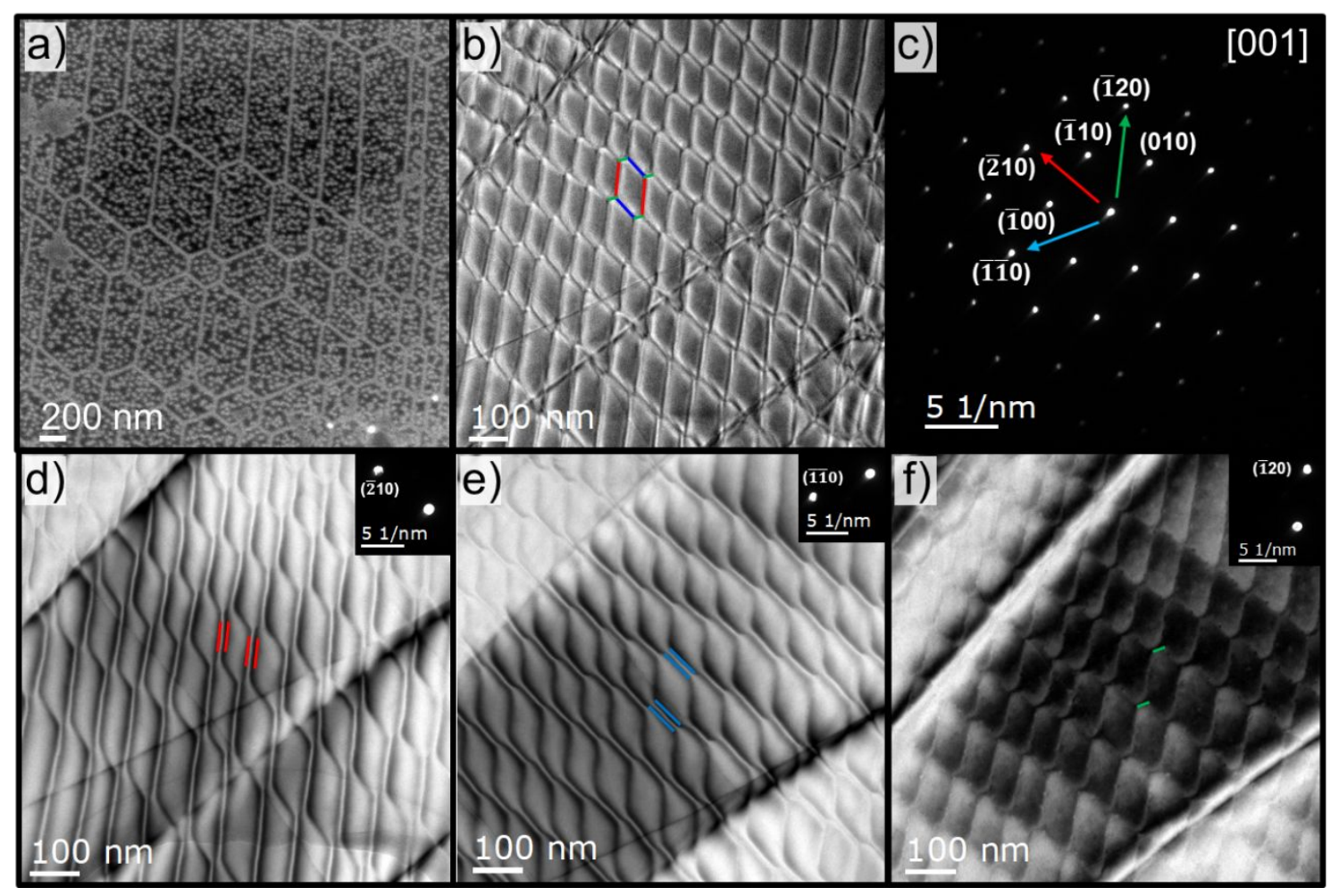

Figure 4. (a) SEM image of a $\mathrm{TiO}_{2}$ deposition pattern in the shape of line networks on mechanically exfoliated $\mathrm{MoS}_{2}$ observed after ALD. (b-c) TEM image and corresponding SAED pattern for a $\mathrm{MoS}_{2}$ flake oriented along the [001] zone axis. Corresponding twobeam condition TEM images for the (d) $(\overline{2} 10),(e)(\overline{1} 10)$, and (f) (120) reciprocal lattice vectors.

The corresponding Burgers vectors $(b)$ for the dislocation lines that spanned the network observed in Figure 4b were characterized by selecting perpendicular reciprocal 
lattice vectors $(g)$ using a two-beam condition. In accordance with the invisibility criterion, the contrast of a dislocation vanishes when $g \bullet b=0$, for dislocation contrast involving a single Bragg reflection. Figure $4 \mathrm{~d}$-f shows that selecting the $g$ vectors $(\overline{2} 10),(\overline{1} \overline{1} 0)$, and $(\overline{1}$ 20) in a two-beam condition results in a contrast doubling for two (Figure $4 d, e$ ) out of three distinct undissociated dislocation lines, instead of the single vanishing contrast expected from the invisibility criterion. Contrast doubling under a two-beam condition is indicative of interactions between dislocations in the network (commonly known as interaction networks; Figure S3) and has been previously observed in naturally occurring $\mathrm{MoS}_{2}$ (molybdenite). ${ }^{[26,27]}$ In interaction networks, two Bragg reflections can be involved in the dislocation contrast, which causes a contrast doubling when selecting perpendicular $g$ vectors. Due to the convolution of the two Bragg reflections their Burgers vectors cannot be definitively isolated.

The three undissociated dislocations observed by TEM meet at a single node, spanning the network observed in Figure $4 \mathrm{~b}$. The line dislocation network can be visualized as ordered corrugations within layers. This corrugation induces a mismatch between layers that can be described as undissociated edge dislocations caused by a buckled region. ${ }^{28]}$ 
Dissociation of a perfect edge dislocation into Shockley partials results in the formation of a stacking fault region bounded by two partials. Consequently, as illustrated in Figure 5 , the relationship between line dislocation networks and triangular networks can be visualized through the dissociation of the three line dislocations, which results in the formation of networks containing triangular extended stacking-fault nodes bounded by partial dislocations (Figure 5), as well as alternating with opposite-facing triangular regions where the stacking is not modified. $[22,29,30]$

The crystal solid can reduce the total stacking fault energy by curving the partial dislocation lines towards the stacking fault region. Consequently, the curvature of the partial dislocation lines depends on the energy of the stacking fault. Figure $5 \mathrm{c}$ shows how the stacking fault energy affects the curvature $(R)$ of the partial dislocation lines and the size of the stacking fault node. ${ }^{[31,32]}$ For a stacking fault energy equal to zero, the partial dislocation lines have zero curvature resulting in a pattern composed of perfectly equilateral triangular nodes. For stacking fault energies greater than zero the partial dislocation lines curve inwards and the area of the stacking fault node gets smaller until 


\begin{abstract}
it eventually collapses into the perfect dislocation lines at sufficiently high stacking fault energies.
\end{abstract}

Figure 5d-g shows SEM images of deposition networks composed of lines, concave triangles connected by lines, corner-sharing concave triangles, and corner-sharing perfectly equilateral triangles on exfoliated $\mathrm{MoS}_{2}$. Triangles with concave side walls were observed for both the triangular deposits and the opposite facing triangular voids, indicating that in some cases the stacking fault node appears to be less reactive than the pristine surface. Several types of stacking faults involving two to three types of stacking alignments have been theoretically identified for molybdenite, ${ }^{[26]}$ but the electronic structure for multilayer disruptions has not been studied extensively. In bilayer 2D stacks, extended triangularly shaped stacking fault networks have recently been shown to exhibit unique electronic structure and properties stemming from the modified density of states (DOS) induced by the altered stacking registry. ${ }^{[28,33]}$ Further understanding the density of states for stacking faults on $2 \mathrm{D}$ materials would provide additional insight into the reactivity effects due to these DOS differences. 
In general, the observed morphologies highlighted in Figure $5 \mathrm{~d}-\mathrm{g}$ provide a direct connection and morphological relationship between the observed line and triangular deposits, and indicate that both types of deposition patterns originate from the same type of interacting dislocation defect network. Hence, the networked triangular deposition pattern observed after ALD on the basal surface of $2 \mathrm{D}$ materials can be explained as a result of preferential seeding induced by the presence of extended triangular stackingfault nodes and pristine opposite-facing surfaces under low supersaturation conditions.
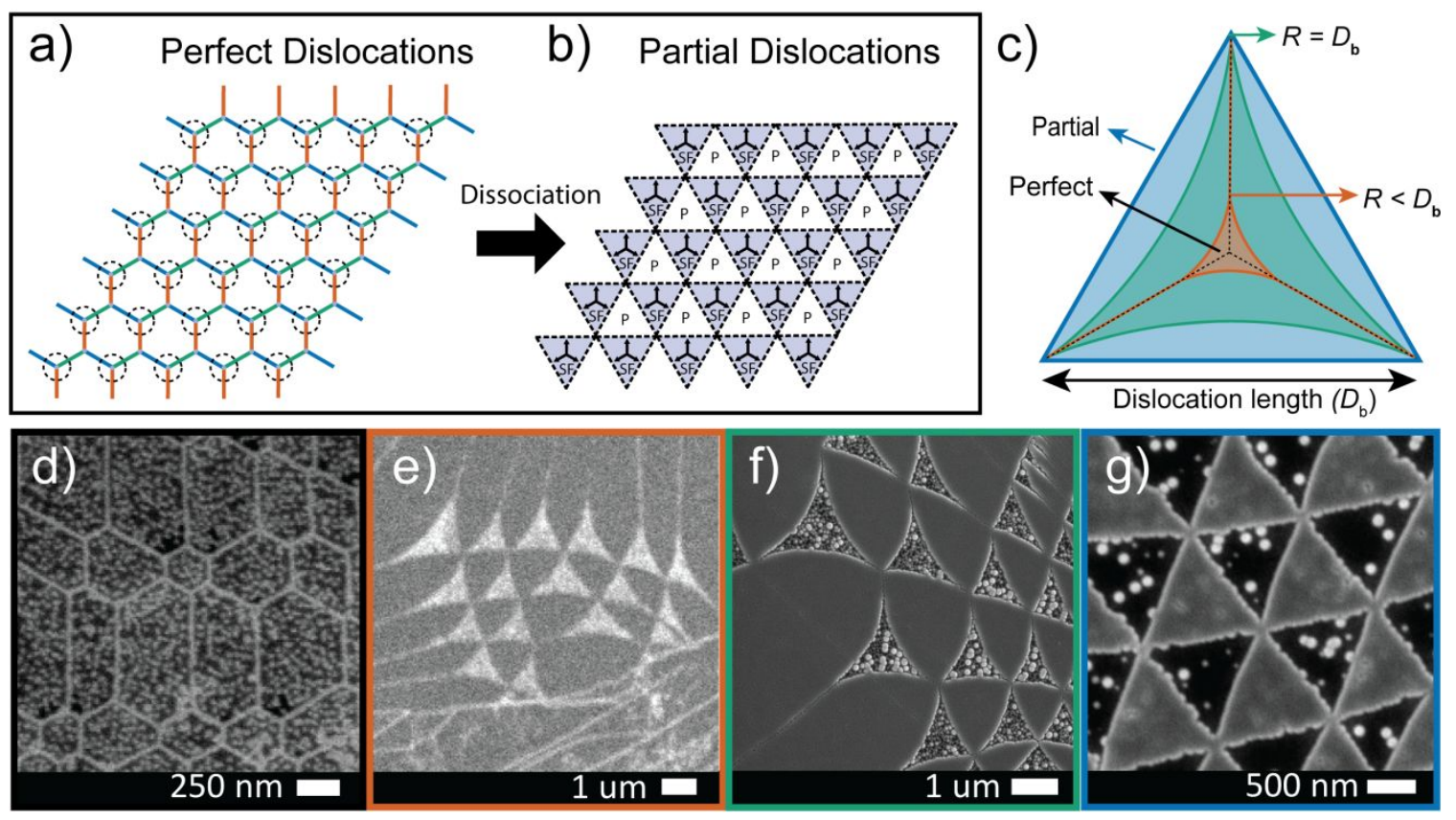

Figure 5. (a) Schematic of dislocation network highlighting the nodes that undergo extension after dissociation of the dislocations into Shockley partials. (b) Network of 
extended stacking-fault (SF) node regions (shaded triangles) composed of alternating SF triangles and opposite-facing triangles composed of pristine $(P)$ surface (unshaded triangles). (c) Schematic showing that higher SF surface energies result in a decrease in the radius of curvature $(R)$ of the dissociated partial dislocations and a decrease in the relative size of the triangular node. Note that in the schematics the three distinct dislocations were assumed to be of equal length $\left(D_{b}\right)$. SEM images of $(d)$ line deposits, (e-f) triangular deposits and empty triangular voids with curved side walls, (f) and equilateral triangular deposits on exfoliated $\mathrm{MoS}_{2}$.

CONCLUSIONS. Highly ordered network patterns, consisting of triangular metal oxide deposits that share corners and orientation, are produced by ALD film growth on the basal surface of mechanically exfoliated 2D layered materials. These triangular patterns persisted across a range of supersaturation reaction conditions and were independent of the position of the substrate in the ALD chamber. The observation of triangular patterns when HOPG or $\mathrm{MoS}_{2}$ was the substrate and when TDMAT or $(\mathrm{EtCp})_{2} \mathrm{Mn}$ was the metal precursor suggests that this type of deposition patterns is intrinsic to the $2 \mathrm{D}$ substrate and 
is independent of precursor chemistry. The observation of undissociated dislocation networks prior to ALD as well as line network deposits after ALD is consistent with the hypothesis that basal plane defects networks, such as periodic dislocation lines and triangularly shaped extended stacking-fault node regions, introduce a surface reactivity landscape that can be exploited under a range of supersaturation conditions to produced patterned deposits. Because the observed type of dislocation networks stems from deformations involving only the van der Waals stacking forces between 2D layers, the observed defect-seeded mechanism for ALD is likely to be general for $2 \mathrm{D}$ layered materials.

\title{
ASSOCIATED CONTENT
}

The Supporting Information is available free of charge on the ACS Publications website at http://pubs.acs.org. Detailed experimental methods and materials used, additional SEM, AFM and optical microscopy data to elucidate SEM contrast and film thickness, and additional discussion related to the dislocation network shown in Figure 4.

\author{
AUTHORS INFORMATION \\ Corresponding Author
}


*E-mail: nslewis@caltech.edu. Tel: (626) 395-6335.

Notes

The authors declare no competing financial interest.

\section{ACKNOWLEDGEMENTS}

M.C. acknowledges support from the Ford Foundation under the Postdoctoral Scholar

Fellowship program. M.C. acknowledges support from the National Science Foundation

CCI Solar Fuels Program under Grant No. CHE-1305124. M.F.M, J.D.W. and N.S.L.

acknowledge support from the U. S. Department of Energy under award DE-FG02-

03ER15483. A.C.T. acknowledges support from the Graduate Research Fellowship

Program of the National Science Foundation. Research was in part carried out at the

Molecular Materials Research Center in the Beckman Institute at the California Institute of Technology.

\section{REFERENCES:}

1. Mak, K. F.; Lee, C.; Hone, J.; Shan, J.; Heinz, T. F. Atomically Thin $\mathrm{MoS}_{2}$ : A New Direct-Gap Semiconductor. Physical Review Letters 2010, 105(13), 136805. 
2. Velický, M.; Bissett, M. A.; Woods, C. R.; Toth, P. S.; Georgiou, T.; Kinloch, I. A.;

Novoselov, K. S.; Dryfe, R. A. W. Photoelectrochemistry of Pristine Mono- and Few-Layer $\mathrm{MoS}_{2}$. Nano Letters 2016, 16 (3), 2023-2032.

3. Lee, J.-U.; Kim, K.; Han, S.; Ryu, G. H.; Lee, Z.; Cheong, H. Raman Signatures of Polytypism in Molybdenum Disulfide. ACS Nano 2016, 10 (2), 1948-1953.

4. Ding, Q.; Song, B.; Xu, P.; Jin, S. Efficient Electrocatalytic and Photoelectrochemical Hydrogen Generation Using $\mathrm{MoS}_{2}$ and Related Compounds. Chem 2016, 1 (5), 699-726.

5. Jaramillo, T. F.; Jørgensen, K. P.; Bonde, J.; Nielsen, J. H.; Horch, S.; Chorkendorff, I. Identification of Active Edge Sites for Electrochemical $\mathrm{H}_{2}$ Evolution from $\mathrm{MoS}_{2}$ Nanocatalysts. Science 2007, 317 (5834), 100-102.

6. Zhou, J.; Qin, J.; Guo, L.; Zhao, N.; Shi, C.; Liu, E.-z.; He, F.; Ma, L.; Li, J.; He, C. Scalable synthesis of high-quality transition metal dichalcogenide nanosheets and their application as sodium-ion battery anodes. Journal of Materials Chemistry $A$ 2016, 4 (44), $17370-17380$. 
7. Lin, D.; Liu, Y.; Liang, Z.; Lee, H.-W.; Sun, J.; Wang, H.; Yan, K.; Xie, J.; Cui, Y.

Layered Reduced Graphene Oxide with Nanoscale Interlayer Gaps as a Stable Host for Lithium Metal Anodes. Nature Nanotechnology 2016, 11 (7), 626-632.

8. Jariwala, D.; Davoyan, A. R.; Tagliabue, G.; Sherrott, M. C.; Wong, J.; Atwater, H. A. Near-Unity Absorption in van der Waals Semiconductors for Ultrathin Optoelectronics. Nano Letters 2016, 16(9), 5482-5487.

9. Yu, W. J.; Vu, Q. A.; Oh, H.; Nam, H. G.; Zhou, H.; Cha, S.; Kim, J.-Y.; Carvalho, A.; Jeong, M.; Choi, H.; Castro Neto, A.H.; Lee, Y.H.; Duan, X. Unusually Efficient Photocurrent Extraction in Monolayer van Der Waals Heterostructure by Tunnelling through Discretized Barriers. Nature Communications 2016, 7(1), 1-9.

10. Ly, T. H.; Zhao, J.; Keum, D. H.; Deng, Q.; Yu, Z.; Lee, Y. H. Hyperdislocations in van der Waals Layered Materials. Nano Letters 2016, 16 (12), 7807-7813.

11. Bampoulis, P.; Sotthewes, K.; Siekman, M. H.; Zandvliet, H. J. W. Local Conduction in $\mathrm{MoxW}_{1}-\mathrm{xSe} \mathrm{S}_{2}$ : The Role of Stacking Faults, Defects, and Alloying. ACS Applied Materials \& Interfaces 2018, 10 (15), 13218-13225. 
12. Li, Z.; Yan, X.; Tang, Z.; Huo, Z.; Li, G.; Jiao, L.; Liu, L.-M.; Zhang, M.; Luo, J.;

Zhu, J. Direct observation of multiple rotational stacking faults coexisting in freestanding bilayer $\mathrm{MoS}_{2}$. Scientific Reports 2017, 7(1), 8323.

13. Cusati, T.; Fortunelli, A.; Fiori, G.; lannaccone, G. Stacking and interlayer electron transport in $\mathrm{MoS}_{2}$. Physical Review B 2018, $98(11), 115403$.

14. Johnson, R. W.; Hultqvist, A.; Bent, S. F. A brief review of atomic layer deposition: from fundamentals to applications. Materials Today 2014, 17(5), 236-246.

15. Hu, S.; Shaner, M. R.; Beardslee, J. A.; Lichterman, M.; Brunschwig, B. S.; Lewis, N. S. Amorphous $\mathrm{TiO}_{2}$ coatings stabilize $\mathrm{Si}, \mathrm{GaAs}$, and $\mathrm{GaP}$ photoanodes for efficient water oxidation. Science 2014, 344 (6187), 1005.

16. Bobb-Semple, D.; Nardi, K. L.; Draeger, N.; Hausmann, D. M.; Bent, S. F. AreaSelective Atomic Layer Deposition Assisted by Self-Assembled Monolayers: A Comparison of Cu, Co, W, and Ru. Chemistry of Materials 2019, 31 (5), 1635-1645. 
17. Lee, H.-B.-R.; Baeck, S. H.; Jaramillo, T. F.; Bent, S. F. Growth of Pt Nanowires by Atomic Layer Deposition on Highly Ordered Pyrolytic Graphite. Nano Letters 2013, 13 (2), 457-463.

18. Kim, K.; Lee, H.-B.-R.; Johnson, R. W.; Tanskanen, J. T.; Liu, N.; Kim, M.-G.; Pang, C.; Ahn, C.; Bent, S. F.; Bao, Z. Selective metal deposition at graphene line defects by atomic layer deposition. Nature Communications 2014, 5(1), 4781.

19. Abendroth, B.; Moebus, T.; Rentrop, S.; Strohmeyer, R.; Vinnichenko, M.; Weling,

T.; Stöcker, H.; Meyer, D. C. Atomic Layer Deposition of $\mathrm{TiO}_{2}$ from Tetrakis(Dimethylamino)Titanium and $\mathrm{H}_{2} \mathrm{O}$. Thin Solid Films 2013, 545, 176-182.

20. Walter, T. N.; Lee, S.; Zhang, X.; Chubarov, M.; Redwing, J. M.; Jackson, T. N.; Mohney, S. E. Atomic Layer Deposition of $\mathrm{ZnO}$ on $\mathrm{MoS}_{2}$ and $\mathrm{WSe}_{2}$. Applied Surface Science 2019, 480, 43-51.

\author{
21. McDonnell, S.; Brennan, B.; Azcatl, A.; Lu, N.; Dong, H.; Buie, C.; Kim, J.; Hinkle, \\ C. L.; Kim, M. J.; Wallace, R. M. $\mathrm{HfO}_{2}$ on $\mathrm{MoS}_{2}$ by Atomic Layer Deposition: Adsorption \\ Mechanisms and Thickness Scalability. ACS Nano 2013, 7(11), 10354-10361.
}


22. Hattendorf, S.; Georgi, A.; Liebmann, M.; Morgenstern, M. Networks of ABA and ABC Stacked Graphene on Mica Observed by Scanning Tunneling Microscopy. Surface Science 2013, 610, 53-58.

23. Shearer, M. J.; Samad, L.; Zhang, Y.; Zhao, Y.; Puretzky, A.; Eliceiri, K. W.; Wright, J. C.; Hamers, R. J.; Jin, S. Complex and Noncentrosymmetric Stacking of Layered Metal Dichalcogenide Materials Created by Screw Dislocations. J. Am. Chem. Soc. 2017, 139 (9), 3496-3504.

24. Arovas, D. P.; Guinea, F. Stacking Faults, Bound States, and Quantum Hall Plateaus in Crystalline Graphite. Physical Review B 2008, 78(24), 245416.

25. Xie, J.; Liao, L.; Gong, Y.; Li, Y.; Shi, F.; Pei, A.; Sun, J.; Zhang, R.; Kong, B.; Subbaraman, R.; Christensen, J.; Cui, Y. Stitching H-BN by Atomic Layer Deposition of LiF as a Stable Interface for Lithium Metal Anode. Science Advances 2017, 3 (11), eaao2170.

26. Hörl, E. M. Dislocations in Molybdenite. Journal of Applied Physics 1965, 36 (1), 253-261. 
27. Kamiya, Y.; Ando, K.; Nonoyama, M.; Uyeda, R. Dislocation Networks and Moiré Patterns of Molybdenite. J. Phys. Soc. Jpn. 1960, 15(11), 2025-2035.

28. Butz, B.; Dolle, C.; Niekiel, F.; Weber, K.; Waldmann, D.; Weber, H. B.; Meyer, B.; Spiecker, E. Dislocations in Bilayer Graphene. Nature 2014, 505 (7484), 533-537.

29. Takahashi, N.; Shiojiri, M.; Enomoto, S. High Resolution Transmission Electron Microscope Observation of Stacking Faults of Molybdenum Disulphide in Relation to Lubrication. Wear 1991, 146(1), 107-123.

30. Delavignette, P.; Amelinckx, S. Dislocation Patterns in Graphite. Journal of Nuclear Materials 1962, 5(1), 17-66

31. Yadav, S. K.; Shao, S.; Wang, J.; Liu, X.-Y. Structural Modifications Due to Interface Chemistry at Metal-Nitride Interfaces. Scientific Reports 2015, 5(1), 17380.

32. Dai, S.; Xiang, Y.; Srolovitz, D. J. Structure and Energy of (111) Low-Angle Twist Boundaries in Al, Cu and Ni. Acta Materialia 2013, 61 (4), 1327-1337. 
33. Alden, J. S.; Tsen, A. W.; Huang, P. Y.; Hovden, R.; Brown, L.; Park, J.; Muller, D. A.; McEuen, P. L. Strain Solitons and Topological Defects in Bilayer Graphene. Proc. Natl. Acad. Sci. USA 2013, $110(28), 11256$.

\section{For Table of Contents Only}

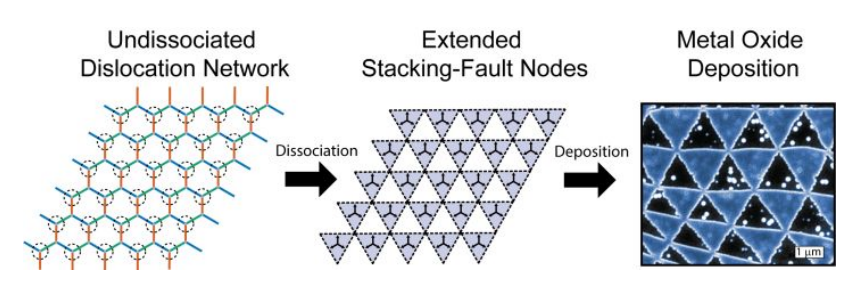

